\title{
Editorial
}

\section{Socio-economic inequalities in diet and body weight: evidence, causes and intervention options}

Diets low in fruits, vegetables and whole grains, and high in saturated fat, salt and sugar, are the major contributors to the burden of chronic diseases globally ${ }^{(1)}$. Previous research, and studies in this issue of Public Health Nutrition, show that unhealthy diets are more commonly observed among socio-economically disadvantaged groups and are key contributors to their higher rates of chronic disease ${ }^{(2-4)}$.

Most research examining socio-economic inequalities in diet and body weight has been descriptive and has focused on identifying the nature, extent and direction of the inequalities. These types of studies are clearly necessary and important. We need, however, to move beyond description of the problem and focus much more on the question of why inequalities in diet and body weight exist. Furthering our understanding of this question will provide the necessary evidence base to develop effective interventions to reduce the inequalities. The challenge of tackling dietary inequalities, however, does not finish here: a maximally effective approach will also require equity-based policies that address the unequal population distribution of social and economic resources, which is the fundamental rootcause of dietary and body weight inequalities.

\section{Current evidence on socio-economic inequalities in diet and body weight}

Studies examining the association between socio-economic position (SEP) and diet have predominantly focused on working-aged adult populations in high-income countries. This research finds that low socio-economic groups are less likely to consume fruits and vegetables, fish, low-fat dairy products and whole grains, and more likely to consume red and processed meat and fast food ${ }^{(5-11)}$. Socio-economically disadvantaged adults in high-income countries also have lower intakes of important micronutrients including folate, vitamin $\mathrm{C}$, vitamin $\mathrm{D}, \mathrm{Ca}, \mathrm{Fe}, \mathrm{Cu}$ and $\mathrm{Se}^{(12)}$, although there is limited evidence that SEP is associated with total energy or macronutrient intake ${ }^{(7,8)}$. Studies describing associations between SEP and diet among children, adolescents and the elderly in high-income countries also show that individuals from disadvantaged backgrounds are more likely to have food and micronutrient intakes that are least consistent with dietary guidelines or reference intakes ${ }^{(12,13)}$.

In this issue of Public Health Nutrition, four studies examine the association between SEP and diet in highincome countries. Ahmadi et al. ${ }^{(14)}$ found that during the school day, Canadian children (aged 10-15 years) with less educated parents were less likely to consume vegetables and more likely to consume sugar-sweetened beverages. Manios et al. ${ }^{(15)}$ show that European children (aged 10-12 years) from low-SEP families less frequently consumed breakfast daily. Khalaf et al. ${ }^{(16)}$ present a complex and mixed picture of the relationship between SEP and dietary habits among female university students (aged 18-25 years) in Saudi Arabia; low parental SEP was associated with both unhealthy and healthy dietary habits, depending on the food type and measure of SEP that was used. According to Dijkstra et al. ${ }^{(17)}$, lower educated Dutch respondents (aged 55-85 years) were less likely to comply with the vegetable guidelines and respondents from lower-income households were less likely to adhere to the fruit and fish guidelines. Taken together, these four studies highlight that dietary inequalities occur at all points across the life course; hence intervention efforts designed to reduce the inequalities should be life-course tailored and targeted accordingly.

Reviews of the association between SEP and body weight in high-income countries ${ }^{(18,19)}$ show that adults from disadvantaged backgrounds are more likely to be overweight or obese, with the relationship being consistently strong and graded for women and somewhat weaker and less consistent for men. Negative associations between SEP and body weight in high-income countries have also been observed in childhood $^{(20,21)}$. Moreover, during the next few decades inequalities in obesity are predicted to widen in some countries ${ }^{(22)}$. In this issue of Public Health Nutrition, overweight/obesity was found to be significantly more likely among socio-economically disadvantaged schoolchildren in Europe (Manios et al. ${ }^{(15)}$ ) and among female university students from less advantaged households in Saudi Arabia (Khalaf et al. ${ }^{(16)}$ ).

The relationship between SEP and diet in low- and middle-income countries has been investigated in a relatively small number of studies. A recent systematic review of thirtythree studies from seventeen low- and middle-income countries concluded that, in general, persons of high SEP have healthier diets, as indicated by higher fruit and vegetable consumption, diet diversity and quality, and higher intakes of vitamin $\mathrm{A}$, vitamin $\mathrm{C}$ and $\mathrm{Fe}^{(23)}$. Socio-economically advantaged groups, however, also have higher intakes of energy, cholesterol and saturated fats and lower intake of fibre. In the current issue, Landais et al. ${ }^{(24)}$ show that Moroccan women of childbearing age from high socio-economic households were significantly more likely 
than their disadvantaged counterparts to consume fruit, and fruit and vegetables combined, and they ate more different types of fruit.

As countries develop economically and shift from being a low- to a middle-income society, they experience a nutrition transition ${ }^{(25,26)}$, characterised by a high consumption of fibre- and grain-rich diets in low-income countries (and higher relative levels of physical activity) moving to increased consumption of sugar, refined grains, animal fat and protein (and lower relative activity) as average wealth of the country grows ${ }^{(23,27)}$. The relationship between SEP and body weight in low- and middleincome countries tends to change in unison with a country's stage in the nutrition transition. In low-income countries, where socio-economically advantaged groups have greater access to energy-dense foods, the association between SEP and body weight is positive for men, women and children. In middle-income countries, where advantaged groups have greater access to more expensive, lowenergy-density, nutrient-rich foods (e.g. fruits, vegetables, unrefined grains), the association between SEP and body weight tends to be negative for women and mixed for men ${ }^{(28)}$. In this issue, Christine et al. ${ }^{(29)}$ provide a withincountry case study of how the association between SEP and body weight changes concomitantly with increases in economic development. Against a backdrop of increasing BMI for both men and women in Argentina between 2005 and 2009, there was an overall inverse graded association between education and BMI for women, which was stronger in geographic regions at higher levels of per capita gross domestic product and weaker at lower levels.

Studies in this issue of Public Health Nutrition that examined associations between SEP, diet and body weight typically investigated these relationships using socioeconomic indicators based on parents' or respondents' education, occupation/employment or income. Importantly, although moderately correlated, measures of SEP are not conceptually interchangeable ${ }^{(6,30)}$ and reflect different pathways linking SEP with diet and body weight ${ }^{(31,32)}$. Future dietary research should continue to investigate these relationships using a range of different socio-economic indicators within the same study ${ }^{(33)}$, as each indicator tells us something different about how and why SEP is related to diet and body weight, and the findings produced by each indicator point to different intervention options.

\section{Explanations for socio-economic inequalities in diet and body weight}

Understanding why socio-economic groups differ in their dietary behaviours and intakes is a necessary precursor to the development and implementation of appropriately designed and targeted interventions, and is integral to their long-term effectiveness. Explaining the existence, persistence and widening of socio-economic inequalities in diet is a complex and challenging task, in part because socioeconomic groups differ on myriad interacting factors that influence their dietary behaviours, food choices and intakes. These factors include (but are not limited to): (i) access to, and capacity to afford, 'healthy' food; (ii) availability of unhealthy food; (iii) access to public and private transport; (iv) neighbourhood safety; (v) social support and peer networks; (vi) time; (vii) adequate income; (viii) knowledge and skills; (ix) beliefs, values, attitudes and motivations; (x) social norms, preferences and habits; (xi) customs, familiarity and tradition; and (xii) perceived capabilities (i.e. self-efficacy). In this issue of Public Health Nutrition, three studies add to this stock of knowledge by undertaking mediation analyses to elucidate the pathways by which SEP is related to diet and body weight. Dijkstra et al. ${ }^{(17)}$ found that taste preference mediated the association between income and adherence to the Dutch dietary guidelines for fruit, while the perception that fish is expensive mediated the association between income and meeting guidelines for fish consumption. Ahmadi et al. ${ }^{(14)}$ observed that socio-economic differences in daily vegetable intake among Canadian schoolaged children were mediated by parental norms; and Manios et $a l .{ }^{(15)}$ found that the relationship between childhood SEP and overweight/obesity was mediated by daily breakfast consumption.

Mediation studies are generating an evidence base that will usefully inform policy and interventions designed to tackle dietary inequalities. To more fully understand and address these inequalities, however, we also require more complex, purpose-designed, multilevel and multidisciplinary dietary research programmes and studies that are conceptualised and conducted from a social determinants of health perspective. This work would be informed by 'real world' frameworks that articulate the complexity and relative contribution of social, economic and environmental factors and life-course processes that are fundamental in differentiating the dietary behaviours and intakes of different socio-economic groups. Ideally, these frameworks will be 'context sensitive' and acknowledge that many individual-level dietary influences (e.g. psychosocial factors) are shaped and circumscribed over time by the lived environments that we are exposed to during everyday life.

\section{Interventions to reduce socio-economic inequalities in diet and body weight}

At present, there is limited evidence to guide policy-making decisions about how to design and implement effective interventions to reduce socio-economic inequalities in diet and body weight. In the context of inequalities' research, intervention effectiveness is usually defined in one of two ways: (i) the intervention results in a greater (healthful) improvement in the dietary outcomes of disadvantaged groups relative to their more advantaged counterparts; or 
(ii) the intervention produces a similar dietary improvement across all socio-economic groups without inadvertently widening the inequalities ${ }^{(34)}$. There are numerous challenges to intervention effectiveness given the large number of interacting factors (operating at multiple levels and across the life course) that differentially influence the diets of socioeconomically advantaged and disadvantaged groups. Arguably, for maximum effectiveness the focus and design of dietary interventions should reflect, or at least be sensitive to, this complexity.

Despite a small intervention evidence base, and heterogeneity in intervention quality, duration and population representativeness (and under-representation of disadvantaged groups in particular), a consensus about the types of interventions likely to be effective in narrowing socio-economic inequalities in diet and obesity is emerging from a number of recent reviews ${ }^{(35,36)}$. In brief, 'agentic' interventions - those which require individuals to make independent choices (e.g. social marketing, food labelling) - are assessed as being less likely to be effective among disadvantaged groups and hence to widen inequalities; by contrast 'structural' interventions - those which involve modifications to environments, contexts, settings or circumstances - are assessed as conferring equal or greater benefit to lower socio-economic groups and therefore unlikely to increase inequalities. Examples include food procurement policies, restriction of junk food advertising to children, taxes on unhealthy food products and healthy school food policies. To date, nutrition interventions have tended to be more agentic than structural $^{(37)}$; hence the possibility exists that some wellintentioned efforts to improve dietary quality across the socio-economic spectrum may have inadvertently widened inequalities, although a number of reviews provide little compelling support for this ${ }^{(38-40)}$.

In two studies in this issue, the authors call for fiscal measures (e.g. subsidies, vouchers, price discounts) as a way of increasing the consumption of fish (Dijkstra et $a l^{(17)}$ ) and fruit (Landais et al. ${ }^{(24)}$ ) among low socio-economic groups. Targeted fiscal measures have been effective in increasing the consumption of nutrient-dense foods (e.g. fruits and vegetables) among low socio-economic groups ${ }^{(41,42)}$ and therefore constitute a potentially effective public health strategy for reducing inequalities in dietary quality ${ }^{(37)}$.

Primavesi et $a l .{ }^{(43)}$ in this issue propose a 'nutrieconomic model' as a method of identifying different food items (e.g. chicken, orange, anchovy, milk) within different food categories (e.g. meat, fruit and vegetables, fish, dairy) on the basis of their nutritional quality per serving and cost. When used to simulate weekly menus, the model was able to demonstrate a $30 \%$ difference (i.e. 35 v. $48 €$ / person per week) in the cost of two equally healthy menus simply by varying the animal protein source. The authors posit that diets of high nutritional quality are not necessarily more expensive, rather the main issue is educating the population (and subgroups within it) about making nutritionally optimal choices. It remains an open question, however, whether the budgets of low-income families are sufficient to afford the cheapest healthy menu, even if nutritional decisions were optimised (which is itself a challenge given their lower levels of dietary knowledge).

\section{Preventing socio-economic inequalities in diet and body weight}

Dietary interventions - ranging from agentic to structural might prove effective in (for example): (i) increasing levels of food and nutrition knowledge among low educated groups; (ii) promoting positive health-enhancing dietary attitudes, preferences and beliefs among unemployed single mothers; (iii) influencing fiscal pricing (taxes and subsidies) that result in low-income families being able to more comfortably afford healthy food; or (iv) changing school canteen policies that restrict access to unhealthy food. Clearly, these outcomes, and the interventions that generate them, are necessary components of any comprehensive (and ideally well-funded) approach to the reduction of socio-economic inequalities in diet and body weight. What these interventions cannot change, however, are the social and economic conditions that are the genesis of the inequalities that the dietary interventions are directed at addressing. So despite the effectiveness of the interventions, the least educated remain less educated, the unemployed continue to seek work, the poor remain poor and those living in disadvantaged neighbourhoods are still at the same address. In short, risk-factor-specific interventions are necessary but not sufficient in our attempts to reduce diet and body weight inequalities.

Fundamentally, social and economic inequalities cause dietary and body weight inequalities; hence, intervention efforts that focus exclusively on diet and body weight irrespective of whether these efforts are agentic or structural - do not change the unequal socio-economic conditions that gave rise to the diet and body weight inequalities. Preventing and reducing socio-economic inequalities in diet and body weight will therefore require the simultaneous implementation of evidence-based intervention efforts targeted at these specific risk factors, in conjunction with progressive social and economic policies that result in a more equitable distribution of the fundamental determinants of health at the societal, neighbourhood, household and individual levels ${ }^{(44)}$.

\section{Acknowledgements}

G.T. is funded by an Australian National Health and Medical Research Council Senior Research Fellowship (\#1003710). S.V. is funded by a fellowship from the National Heart Foundation of New Zealand (\#1614). Thanks go to Dr Belinda Hewitt at the Institute for Social 
Science Research and School of Social Science, The University of Queensland, St Lucia, Australia, and Dr Jerome Rachele at the School of Public Health and Social Work, Queensland University of Technology, Kelvin Grove, Australia, for useful comments on an earlier draft of this editorial.

Gavin Turrell and Stefanie Vandevijvere Associate Editors

\section{References}

1. Lim SS, Vos T, Flaxman AD et al. (2012) A comparative risk assessment of burden of disease and injury attributable to 67 risk factors and risk factor clusters in 21 regions, 1990-2010: a systematic analysis for the Global Burden of Disease Study 2010. Lancet 380, 2224-2260.

2. Davey Smith G \& Brunner E (1997) Socioeconomic differentials in health: the role of nutrition. Proc Nutr Soc 56, 75-90.

3. James WPT, Nelson M, Ralph A et al. (1997) The contribution of nutrition to inequalities in health. BMJ 314, 1545-1549.

4. Martikainen P, Brunner E \& Marmot M (2003) Socioeconomic differences in dietary patterns among middle-aged men and women. Soc Sci Med 56, 1397-1410.

5. De Irala-Estevez J, Groth M, Johansson L et al. (2000) A systematic review of socio-economic differences in food habits in Europe: consumption of fruit and vegetables. Eur J Clin Nutr 54, 706-714.

6. Lallukka T, Laaksonen M, Rahkonen O et al. (2007) Multiple socio-economic circumstances and healthy food habits. Eur J Clin Nutr 61, 701-710.

7. Darmon N \& Drewnowski A (2007) Does social class predict diet quality? Am J Clin Nutr 87, 1107-1117.

8. Giskes K, Avendano M, Brug J et al. (2010) A systematic review of studies on socioeconomic inequalities in dietary intakes associated with weight gain and overweight/obesity conducted among European adults. Obes Rev 11, 413-429.

9. Miura K, Giskes K \& Turrell G (2012) Socio-economic differences in takeaway food consumption among adults. Public Health Nutr 15, 218-226.

10. Thornton LE, Bentley RJ \& Kavanagh AM (2011) Individual and area-level socioeconomic associations with fast food purchasing. J Epidemiol Community Health 65, 873-880.

11. Maguire ER \& Monsivais P (2015) Socio-economic dietary inequalities in UK adults: an updated picture of key food groups and nutrients from national surveillance data. $\mathrm{BrJ}$ Nutr 113, 181-189.

12. Novakovic R, Cavelaars A, Geelen A et al. (2014) Socioeconomic determinants of micronutrient intake and status in Europe: a systematic review. Public Health Nutr 17, 1031-1045.

13. Hanson MD \& Chen E (2007) Socioeconomic status and health behaviors in adolescence: a review of the literature. J Behav Med 30, 263-285.

14. Ahmadi N, Black JL, Velazquez CE et al. (2015) Associations between socio-economic status and school-day dietary intake in a sample of grade 5-8 students in Vancouver, Canada. Public Health Nutr 18, 764-773.

15. Manios Y, Moschonis G, Androutsos O et al. (2015) Family sociodemographic characteristics as correlates of children's breakfast habits and weight status in eight European countries. The ENERGY (EuropeaN Energy balance Research to prevent excessive weight Gain among Youth) project. Public Health Nutr 18, 774-783.

16. Khalaf A, Westergren A, Berggren V et al. (2015) Prevalence and association of female weight status and dietary habits with sociodemographic factors: a cross-sectional study in Saudi Arabia. Public Health Nutr 18, 784-796.
17. Dijkstra SC, Neter JE, van Stralen MM et al. (2015) The role of perceived barriers in explaining socio-economic status differences in adherence to the fruit, vegetable, and fish guidelines in older adults: a mediation study. Public Health Nutr 18, 797-808.

18. McLaren L (2007) Socioeconomic status and obesity. Epidemiol Rev 29, 29-48.

19. Rokholm B, Baker JL \& Sorensen TIA (2010) The levelling off of the obesity epidemic since the year 1999 - a review of evidence and perspectives. Obes Rev 11, 835-846.

20. Shrewsbury V, Wardle J 2008) Socioeconomic status and adiposity in childhood: a systematic review of crosssectional studies 1990-2005. Obesity (Silver Spring) 16, 275-284.

21. Wang Y \& Lim H (2012) The global childhood obesity epidemic and the association between socio-economic status and childhood obesity. Int Rev Psychiatry 24, 176-188.

22. Backholer K, Mannan H, Magliano D et al. (2012) Projected socioeconomic disparities in the prevalence of obesity amongst Australian adults. Aust $N Z J$ Public Health 36, 557-563.

23. Mayen A-L, Marques-Vidal P, Paccaud F et al. (2014) Socioeconomic determinants of dietary patterns in low- and middle-income countries: a systematic review. Am J Clin Nutr 100, 1520-1531.

24. Landais E, Bour A, Gartner A et al. (2015) Socio-economic and behavioural determinants of fruit and vegetable intake in Moroccan women. Public Health Nutr 18, 809-816.

25. Popkin BM \& Gordon-Larsen P (2004) The nutrition transition: worldwide obesity dynamics and their determinants. Int J Obes Rel Metab Disord 28, Suppl. 3, S2-S9.

26. Popkin BM (2006) Global nutrition dynamics: the world is shifting rapidly toward a diet linked with noncommunicable diseases. Am J Clin Nutr 84, 289-298.

27. Malik VS, Willett WC \& Hu FB (2013) Global obesity: trends, risk factors, and policy implications. Nat Rev Endocrinol 9 , $13-27$.

28. Dinsa GD, Goryakin Y, Fumagalli E et al. (2012) Obesity and socioeconomic status in developing countries: as systematic review. Obes Rev 13, 1067-1079.

29. Christine PJ, Diez Roux AV, Wing JJ et al. (2015) Temporal trends in BMI in Argentina by socio-economic position and province-level economic development, 2005-2009. Public Health Nutr 18, 817-826.

30. Geyer S, Hernstom O, Peter R et al. (2006) Education, income and occupational class cannot be used interchangeably in social epidemiology. Empirical evidence against a common practice. J Epidemiol Community Health 60, 804-810.

31. Galobardes B, Lynch J \& Smith GD (2007) Measuring socioeconomic position in health research. Br Med Bull 81-82, 21-37.

32. Turrell G, Hewitt B, Patterson C et al. (2003) Measuring socio-economic position in dietary research: is choice of socio-economic indicator important? Public Health Nutr $\mathbf{6}$, 191-200.

33. Braveman PA, Cubbin C, Egerter S et al. (2005) Socioeconomic status in health research: one size does not fit all. JAMA 294, 2879-2888.

34. Kristjansson EA, Robinson V, Petticrew M et al. (2007) School feeding for improving the physical and psychological health of disadvantaged elementary school children. Cochrane Database Syst Rev issue 1, CD004676.

35. Beauchamp A, Backholer K, Magliano D et al. (2014) The effect of obesity prevention interventions according to socioeconomic position: a systematic review. Obes Rev $\mathbf{1 5}$, 541-554.

36. Backholer K, Beauchamp A, Ball K et al. (2014) A framework for evaluating the impact of obesity prevention strategies on socioeconomic inequalities in weight. Am J Public Health 104, e43-e50. 
37. Ni Mhurchu C (2010) Food costs and healthful diets: the need for solution-oriented research and policies. Am J Clin Nutr 92, 1007-1008.

38. Oldroyd J, Burns C, Lucas P et al. (2008) The effectiveness of nutrition interventions on dietary outcomes by relative social disadvantage: a systematic review. J Epidemiol Community Health 62, 573-579.

39. Hillier-Brown FC, Bambra CL, Cairns J-M et al. (2014) A systematic review of the effectiveness of individual, community and societal-level interventions at reducing socioeconomic inequalities in obesity among adults. Int $J$ Obes (Lond) 38, 1483-1490.

40. Hillier-Brown FC, Bambra CL, Cairns J-M et al. (2014) A systematic review of the effectiveness of individual, community, and societal-level interventions at reducing socioeconomic inequalities in obesity amongst children. BMC Public Health 14, 834.
41. Geliebter A, Ang IYH, Bernales-Korins M et al. (2013) Supermarket discounts of low-energy density foods: effects on purchasing, food intake, and bodyweight. Obesity (Silver Spring) 21, e 542-e548.

42. Waterlander WE, de Boer MR, Schuit AJ et al. (2013) Price discounts significantly enhance fruit and vegetable purchases when combined with nutrition education: a randomized controlled supermarket trial. Am J Clin Nutr $\mathbf{9 7}$, 886-895.

43. Primavesi L, Caccavelli G, Ciliberto A et al. (2015) Nutrieconomic model can facilitate healthy and low-cost food choices. Public Health Nutr 18, 827-835.

44. Loring B \& Robertson A (2014) Obesity and Inequities. Guidance for Addressing Inequities in Overweight and Obesity. Copenhagen: WHO Regional Office for Europe; available at http://www.euro.who.int/_data/assets/pdf_ file/0003/247638/obesity-090514.pdf?ua=1 